\title{
Capsaicin, A Ligand for Vanilloid Receptor-1, Transduces Suppressive Signal for Osteoclast Differentiation in Bone
}

\author{
Morichika Takita, Masaki Inada, and Chisato Miyaura* \\ Department of Biotechnology and Life Science, Tokyo University of Agriculture and Technology, 2-24-16 Nakamachi, Koganei, \\ Tokyo 184-8588, Japan
}

(Received November 29, 2006; Accepted December 12, 2006; Published online December 19, 2006)

Vanilloid receptor-1 (VR1) has been reported to exhibit multiple functions which can transduce painsensitive signals in nerve systems. A VR1 ligand, capsaicin, has been reported to show activities against inflammation and cancer growth, however, its role in bone metabolism is still unknown. Here, we examined the effect of capsaicin on cytokine-induced inflammatory bone resorption. Capsaicin suppressed interleukin-1-induced bone resorption in a mouse calvarial organ ex vivo culture in a dose-dependent manner. An assay using cocultures of osteoblasts and bone marrow cells clearly showed the inhibition of osteoclast formation by treatment with capsaicin. Receptor activator of NF- $\kappa$ B ligand (RANKL), the sole inducer of osteoclast formation, is known to be produced by osteoblasts. In the cocultures of bone marrow cells and osteoblasts, the expression of RANKL was suppressed by capsaicin. VR1 showed expression predominantly in osteoblasts, suggesting that capsaicin directly modulates osteoclast differentiation through the suppression of RANKL expression. VR1 ligands like capsaicin have the potential for use as clinical drugs targeting some bone diseases involving cytokine-induced bone resorption.

Key words_ - vanilloid receptor-1, bone, capsaicin, osteoclast formation, bone resorption

\section{INTRODUCTION}

Skeletal diseases like osteoporosis, osteoarthritis, and periodontitis are common diseases, which are associated with severe bone loss. Current innovations in clinical drug development for these

\footnotetext{
*To whom correspondence should be addressed: Department of Biotechnology and Life Science, Tokyo University of Agriculture and Technology, 2-24-16 Nakamachi, Koganei, Tokyo 184-8588, Japan. Tel.: +81-42-388-7390; Fax: +81-42-3887390; E-mail: miyaura@cc.tuat.ac.jp
}

diseases are mainly focused on the suppression of bone resorption by anti-resorptive drugs such as calcitonin and bisphosphonates. ${ }^{1,2)}$ These drugs have been known to act directly on mature osteoclasts, and to destruct osteoclast-featured cytoskeleton, called actin-rings formed by actin fibers. Previously, the definitive molecule of osteoclast differentiation was cloned and named receptor activator of $\mathrm{NF}-\kappa \mathrm{B}$ ligand (RANKL). RANKL is expressed predominantly in osteoblasts and regulates osteoclast differentiation. Osteoblasts respond to various inflammatory factors such as interleukin (IL)-1, IL-6, and prostaglandin $\mathrm{E}_{2}\left(\mathrm{PGE}_{2}\right)$, and RANKL production was induced to regulate osteoclast differentiation and functions. ${ }^{3-5)}$ IL-1 is a powerful mediator of inflammatory bone resorption in various diseases through its association with increased RANKL expression in osteoblasts.

Recently, several groups have reported the feature of vanilloid receptors (VRs/TRPVs), consist of six different receptors called TRPV1 (VR1), TRPV2 (VRL-1), TRPV3, TRPV4, TRPM8, and TRPA1, most of which were thermal-sensitive molecules. ${ }^{6-8)}$ Since VR1 has been cloned from a cDNA library of the dorsal root ganglion in sensory neurons, the VR1 signal has been suggested as a potential regulator of pain associated with thermal stimulation. $\left.{ }^{9}\right)$ Capsaicin, a typical ligand for VR1, is derived from chilly peppers, which elicit a burning sensation through VR1. ${ }^{10)}$ The following study also suggested that the neuronal stimulation had a relationship with bone remodeling. ${ }^{11)}$ Sympathectomy and capsaicin treatment showed resemble effects on osteoclast formation in rat neonatal and adult jaws, ${ }^{11,12)}$ however, the molecular basis of the regulation of osteoclast differentiation through VR1 is still unknown. Here, we examined the molecular action of capsaicin in osteoclast differentiation and bone resorption. 


\section{MATERIALS AND METHODS}

Animals and Drugs — Newborn (2-day-old) and adult (6-week-old) $d d y$ mice were obtained from Japan SLC Inc. (Shizuoka, Japan). Recombinant human IL- $1 \alpha$ was purchased from R\&D Systems (Minneapolis, MN, U.S.A.). Capsaicin was purchased from Wako Pure Chemical Industries (Osaka, Japan). All other chemicals were of analytical grade.

Mouse Calvarial Culture — Two-day-old mice were sacrificed and their calvariae were aseptically harvested and dissected free of tissues. The calvariae were divided into halves and cultured for $24 \mathrm{hr}$ at $37^{\circ} \mathrm{C}$ under $5 \% \mathrm{CO}_{2}$ in air in $0.4 \mathrm{ml}$ of BGJb medium (Invitrogen, Carlsbad, CA, U.S.A.) containing $1 \mathrm{mg} / \mathrm{ml}$ bovine serum albumin (fraction V, Sigma, St. Louis, MO, U.S.A.). After pre-culture for $24 \mathrm{hr}$, each half calvaria was transferred to fresh medium, with and without IL-1 $(2 \mathrm{ng} / \mathrm{ml})$, and cultured for an additional 5 days. To determine the bone-resorbing activity of test materials, the concentration of calcium in the conditioned medium was measured on day 5 using the o-cresolphthalein complexon (OCPC) method. ${ }^{13)}$

Culture of Primary Mouse Osteoblastic Cells Primary osteoblastic cells were isolated from 2-dayold mouse calvariae after five routine sequential digestions with $0.1 \%$ collagenase (Roche Applied Science) and $0.2 \%$ dispase (Roche Diagnostics, Mannheim, Germany), as described previously. ${ }^{13)}$ Osteoblastic cells collected from fractions 2 to 5 were combined and cultured in minimum essential medium alpha ( $\alpha$ MEM) (Invitrogen) supplemented with $10 \%$ fetal calf serum (FCS) at $37^{\circ} \mathrm{C}$ under $5 \%$ $\mathrm{CO}_{2}$ in air.

Osteoclast Formation in Cocultures of Mouse Bone Marrow Cells and Osteoblasts - Bone marrow cells $\left(3 \times 10^{6}\right.$ cells $)$ were isolated from 6week-old mice and cocultured with the primary osteoblastic cells $\left(1 \times 10^{4}\right.$ cells $)$ in $1 \mathrm{ml}$ of $\alpha \mathrm{MEM}$ containing $10 \%$ FCS on 24 -well plates. The cultures were fed every 3 days by replacing $0.9 \mathrm{ml}$ of the old medium with fresh medium. After being cultured for 7 days, the cells adhering to the well surface were stained for tartrate-resistant acid phosphatase (TRAP). TRAP-positive multinucleated cells containing three or more nuclei per cell were counted as osteoclasts. To extract total RNA for Reverse transcriptase (RT)-PCR analysis, the cells adhering to the well surface were lysed on day 7 .
RT-PCR Analysis — To obtain bone marrow macrophages, mouse bone marrow cells were cultured for 7 days in the presence of $100 \mathrm{ng} / \mathrm{ml}$ of macrophage colony stimulating factor (MCSF). Total RNA was extracted from cultured cells and mouse brain, using the acid guanidium-phenolchloroform method. ${ }^{13)}$ cDNA was synthesized from $5 \mu \mathrm{g}$ of total RNA by reverse transcriptase (Superscript II Pre-amplification System, Invitrogen) and amplified via PCR. The primers in PCR for the mouse RANKL, mouse VR1, and mouse glyceraldehyde-3-phosphate dehydrogenase (GAPDH) genes were used as reported previously. ${ }^{13,14)}$ The PCR product was run on a $1.5 \%$ agarose gel and stained with ethidium bromide.

Statistical Analysis — The data are expressed as the means \pm SEM. The significance of differences was analyzed using Student's $t$-test.

\section{RESULTS}

\section{Capsaicin Inhibited IL-1-Induced Bone Resorp- tion in Calvarial Ex Vivo Culture}

Capsaicin is a major ingredient of temperature stimulation in hot chilly peppers, the structure with olvanyl-flame being shown in Fig. 1. The binding affinity to VR1 was reported, ${ }^{9)}$ and the following experiments were guided by the concentrations of capsaicin as the VR1 agonist. We first examined whether capsaicin has a protective effect against bone resorption in ex vivo organ culture. Bone-resorbing activity was monitored by the increase in medium calcium, which is significantly elevated by treatment with bone-resorbing factors. In the organ culture, IL-1 greatly stimulated boneresorbing activity compared to vehicle treatment. The treatment with $3-30 \mu \mathrm{M}$ capsaicin in the presence of IL-1 dose-dependently suppressed boneresorbing activities induced by IL-1 (Fig. 2). Since IL-1 is a typical bone-resorbing cytokine produced in the state of inflammation, capsaicin may act as an anti-inflammatory factor, which prevents cytokineinduced bone loss.

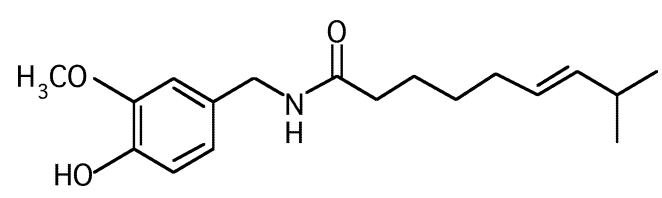

Fig. 1. Chemical Structure of Capsaicin 


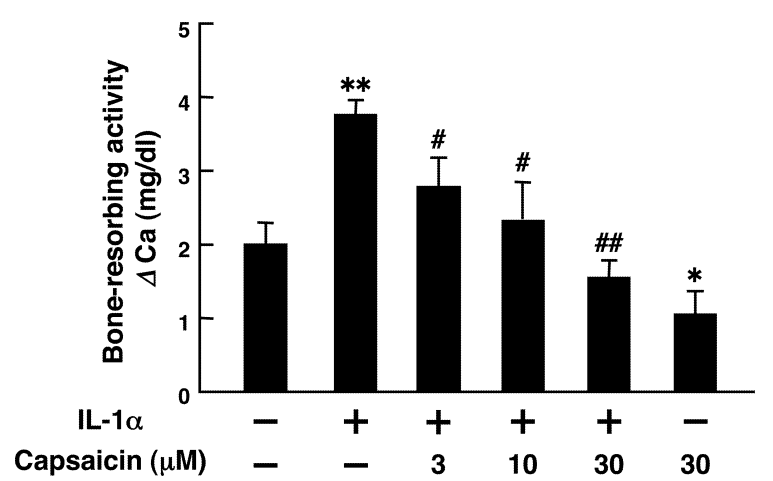

Fig. 2. Capsaicin Suppresses Bone-Resorbing Activity in Mouse Calvarial Cultures

Calvariae collected from 2-day-old mice were cultured for 5 days with $2 \mathrm{ng} / \mathrm{ml}$ of IL- $1 \alpha$ and/or various concentrations of capsaicin (3$30 \mu \mathrm{M})$. Conditioned medium was collected and the calcium content was measured. Bone-resorbing activity was expressed as the increase in the medium calcium. Significantly different, ${ }^{*} p<0.05,{ }^{* *} p<0.001$ versus control, ${ }^{\#} p<0.05,{ }^{\# \#} p<0.001$ versus IL- $1 \alpha$. Data are expressed as the means \pm SEM of three to five cultures.

\section{Effect of Capsaicin on Osteoclast Formation In- duced by IL-1}

To further understand the mechanisms of capsaicin on IL-1-induced bone resorption, we performed osteoclast formation analysis by mixed coculture using primary osteoblasts and bone marrow cells in the presence of IL-1 and capsaicin. By treatment with IL-1, osteoclast maker, TRAPpositive multinucleated osteoclasts were formed on day 7 in culture. Simultaneous treatment with $3 \mu \mathrm{M}$ capsaicin and IL-1 led to a significant inhibition of osteoclast formation, and $30 \mu \mathrm{M}$ capsaicin completely attenuated the IL-1-induced osteoclast formation (Fig. 3A). Several possibilities were counted on the mechanism, e.g., capsaicin has a direct action on osteoclasts, osteoclast precursors or osteoblasts. Then, we examined the levels of RANKL expression after treatment with IL-1 and capsaicin in a coculture system.

\section{Effect of Capsaicin on IL-1-Induced RANKL Expression in Coculture System}

In cocultures of bone marrow cells and osteoblasts, IL-1 clearly induced mRNA expression of RANKL that was coordinating to the number of formed TRAP-positive osteoclasts. In the presence of capsaicin $(30 \mu \mathrm{M})$ perfectly inhibited IL1-induced RANKL mRNA expression (Fig. 3B), which is consistent with the suppression of IL-1induced osteoclast formation by capsaicin shown in Fig. 3A. These results suggest that capsaicin, a VR1 agonist, inhibits osteoclast formation by suppress-
A
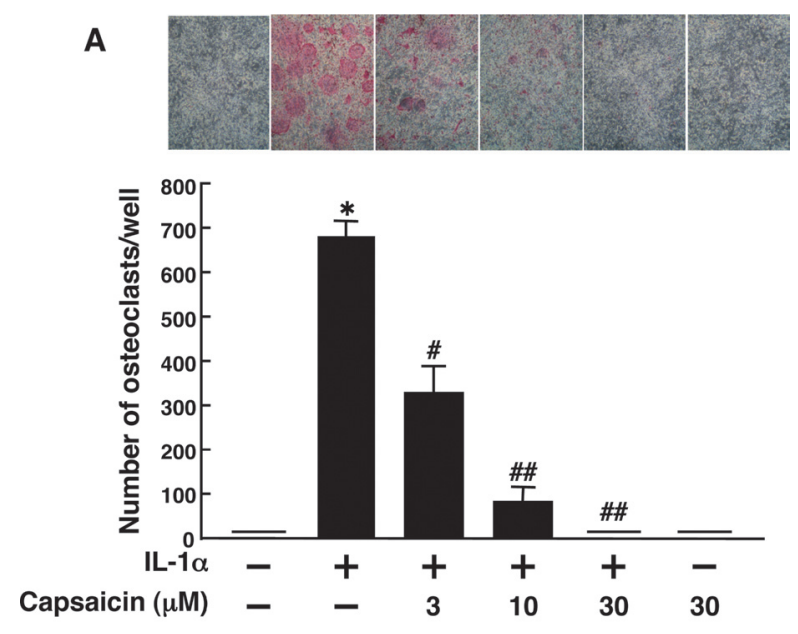

B

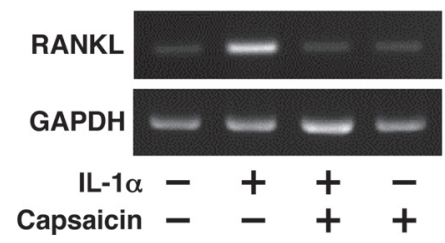

Fig. 3. Capsaicin Inhibits Osteoclast Formation Induced by IL$1 \alpha$ in Coculture of Mouse Bone Marrow Cells and Osteoblasts

Mouse bone marrow cells $\left(3 \times 10^{6}\right.$ cells $)$ and osteoblasts $\left(1 \times 10^{4}\right.$ cells) were cocultured for 7 days in the presence or absence of $2 \mathrm{ng} / \mathrm{ml}$ IL- $1 \alpha$ or $3-30 \mu \mathrm{M}$ capsaicin. (A) Representative fields of TRAP staining are shown (upper panel). The number of osteoclasts was counted (lower panel). Significantly different, ${ }^{*} p<0.001$ versus control, ${ }^{\#} p<0.01$, \#\# $p<0.001$ versus IL- $1 \alpha$. Data are expressed as the means \pm SEM of four wells. (B) The expression of RANKL mRNA was examined by RT-PCR in coculture of mouse bone marrow cells and osteoblasts. Mouse bone marrow cells and osteoblasts were cocultured for 7 days in the presence or absence of $2 \mathrm{ng} / \mathrm{ml}$ of IL- 1 and/or $30 \mu \mathrm{M}$ capsaicin, and total RNA was extracted from adherent cells. The expression of RANKL mRNA was analyzed by RT-PCR.

ing RANKL expression. It is certain that osteoblasts are a predominant source of RANKL expression in bone. We next examined whether VR1 is expressed in osteoblasts.

\section{VR1 Expression in Osteoclast-supportive Os- teoblasts}

To examine the expression of VR1 in osteoblasts, we obtained primary osteoblasts from calvariae that were used in mixed cocultures to examine osteoclast formation. The osteoblastic cell line MC3T3-E1 was also subjected to this experiment, because the cell line has a capacity to support osteoclast formation in the presence of IL-1. Total RNA was isolated from respective cells, and applied to RT-PCR using the primers of mouse VR1. The data clearly showed the expression of VR1 mRNA in both primary osteoblasts and MC3T3-E1 cells 


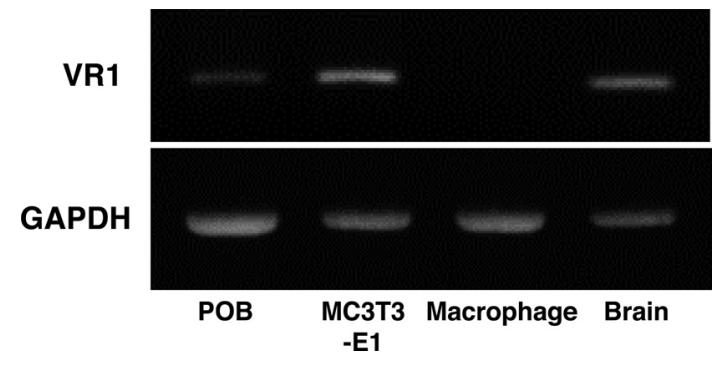

Fig. 4. Expression of VR1 mRNA in Mouse Osteoblasts Mouse primary osteoblasts (POB) and a mouse osteoblastic cell line (MC3T3-E1) were cultured with $10 \%$ FCS in $\alpha$ MEM to cell confluence. To obtain bone marrow macrophages, mouse bone marrow cells were cultured for 7 days in the presence of $100 \mathrm{ng} / \mathrm{ml}$ of MCSF. After the cultures, total RNA was extracted and the expression of VR1 mRNA was analyzed by RT-PCR. Total RNA was also extracted from the mouse brain for RT-PCR, and used as a positive control of VR1 expression.

(Fig. 4). Expression of VR1 mRNA could not be detected in bone marrow macrophages, which are known as osteoclast precursors (Fig. 4). As reported previously, VR1 was clearly detected in the mouse brain. These results suggest that capsaicin binds to VR1 in osteoblasts and suppresses the expression of RANKL induced by IL-1, following the inhibition of osteoclast formation.

\section{DISCUSSION}

Our findings in the present study showed that the VR1 agonist, capsaicin, attenuated boneresorbing activity by the suppression of osteoclast differentiation. Previous studies have focused on capsaicin as a neurotoxin, which induces damage to neurons through VR1 signal, whereby the nervous system modulates osteoclast formation after the local injection of capsaicin. ${ }^{11,12)}$ Calcitonin gene related peptide (CGRP) was produced by sensory neurons, to maintain pain block, and its receptor was found in neuronal tissue. CGRP is an alternatively spliced molecule from the CT/CGRP gene, and known to weakly bind to the calcitonin receptor. It is well known that calcitonin binds to the CT receptor in mature osteoclasts and induces the extinction of osteoclast functions. ${ }^{1)}$ CGRP produced by neuronal cells could bind to the $\mathrm{CT}$ receptor on osteoclast and suppress osteoclast function in vivo. ${ }^{15,16)}$ In the present study, IL-1 induced severe bone resorption in calvarial culture that was associated with increased TRAP-positive osteoclast formation, and capsaicin treatment clearly suppressed osteoclast formation in the organ culture (data not shown). The culture system using calvarial ex vivo explants, which mainly consists of cortical bone, may contain less neuronal tissue, suggesting that the suppressive effect of capsaicin on bone resorption is raised by its action on osteoclasts or osteoblasts.

Osteoclasts are cells originating from hematopoietic stem cells that differentiate into monocyte lineages, and MCSF and RANKL are key modulators for their differentiation into osteoclasts. A recent study indicated the direct action of capsaicin on macrophages. Lipopolysaccharide (LPS)-induced $\mathrm{PGE}_{2}$ production was inhibited in the culture of peritoneal macrophages, however, the macrophages did not possess VR1. ${ }^{17)}$ The following study showed that capsaicin acts on the macrophage cell line RAW 264.7 to attenuate LPSinduced COX-2 expression, but RAW 264.7 cells did not express VR1. ${ }^{18)}$ Since mouse macrophage cells did not express VR1, the non-genomic action of capsaicin could be counted, and further studies will be required to clarify the direct effects of capsaicin on macrophages.

In the present study, we found the expression of VR1 in osteoblasts, a major source of RANKL in bone, suggesting that capsaicin directly bind to osteoblasts and suppress their expression of RANKL. It is not likely that capsaicin affects bone marrow macrophages, the lineage of osteoclast precursors to suppress osteoclast differentiation, because bone marrow macrophages showed no expression of VR1 (Fig.4). Both resiniferatoxin, a natural VR1 agonist, and olvanil, a synthetic VR1 agonist, suppressed osteoclast formation induced by IL1 in cocultures of mouse bone marrow cells and osteoblasts (data not shown). Therefore, the effects of these compounds including capsaicin on osteoclast differentiation may be mediated by the VR1 signal. Recently, the anti-inflammatory effect of capsaicin was reported in a model of intestinal diseases, and the report clearly indicated that capsaicin, a VR1 agonist, has a potential to protect against inflammatory bowel diseases associated with severe inflammation. ${ }^{19)}$ Further studies are need to define a possible application of capsaicin as an anti-inflammatory agent in some diseases. Taken together, we showed that capsaicin inhibited IL-1-induced osteoclast formation and bone resorption, the mechanism of which is mediated by VR1 expressed in osteoblasts to suppress RANKL expression. Therefore, capsaicin holds the considerable potentials for pharmaceutical development in 
the cases associated with inflammatory bone loss.

\section{REFERENCES}

1) Ikegame, M., Ejiri, S. and Ozawa, H. (2004) Calcitonin-induced change in serum calcium levels and its relationship to osteoclast morphology and number of calcitonin receptors. Bone, 35, 27-33.

2) Suzuki, K., Takeyama, S., Sakai, Y., Yamada, S. and Shinoda, H. (2006) Current topics in pharmacological research on bone metabolism: inhibitory effects of bisphosphonates on the differentiation and activity of osteoclasts. J. Pharmacol. Sci., 100, 189-194.

3) Yasuda, H., Shima, N., Nakagawa, N., Yamaguchi, K., Kinosaki, M., Mochizuki, S., Tomoyasu, A., Yano, K., Goto, M., Murakami, A., Tsuda, E., Morinaga, T., Higashio, K., Udagawa, N., Takahashi, N. and Suda, T. (1998) Osteoclast differentiation factor is a ligand for osteoprotegerin/osteoclastogenesis-inhibitory factor and is identical to TRANCE/RANKL. Proc. Natl. Acad. Sci. U.S.A., 31, 3597-3602.

4) Lacey, D. L., Timms, E., Tan, H. L., Kelley, M. J., Dunstan, C. R., Burgess, T., Elliott, R., Colombero, A., Elliott, G., Scully, S., Hsu, H., Sullivan, J., Hawkins, N., Davy, E., Capparelli, C., Eli, A., Qian, Y. X., Kaufman, S., Sarosi, I., Shalhoub, V., Senaldi, G., Guo, J., Delaney, J. and Boyle, W. J. (1998) Osteoprotegerin ligand is a cytokine that regulates osteoclast differentiation and activation. Cell, 17, 165176.

5) Fuller, K., Wong, B., Fox, S., Choi, Y. and Chambers, T. J. (1998) TRANCE is necessary and sufficient for osteoblast-mediated activation of bone resorption in osteoclasts. J. Exp. Med., 188, $997-$ 1001.

6) Clapham, D. E. (2003) TRP channels as cellular sensors. Nature, 426, 517-524.

7) Jordt, S. E., McKemy, D. D. and Julius, D. (2003) Lessons from peppers and peppermint: the molecular logic of thermosensation. Curr. Opin. Neurobiol., 13, 487-492.

8) Patapoutian, A., Peier, A. M., Story, G. M. and Viswanath, V. (2003) Thermo TRP channels and beyond: mechanisms of temperature sensation. Nat. Rev. Neurosci., 4, 529-539.

9) Caterina, J., Schumacher, M. M. A., Tominaga, M., Rosen, T. A., Levine, J. D. and Julius, D. (1997) The capsaicin receptor: a heat-activated ion channel in the pain pathway. Nature, 389, 816-824.

10) Davis, J. B., Gray, J., Gunthorpe, M. J., Hatcher,
J. P., Davey, P. T., Overend, P., Harries, M. H., Latcham, J., Clapham, C., Atkinson, K., Hughes, S. A., Rance, K., Grau, E., Harper, A. J., Pugh, P. L., Rogers, D. C., Bingham, S., Randall, A. and Sheardown, S. A. (2000) Vanilloid receptor-1 is essential for inflammatory thermal hyperalgesia. $\mathrm{Na}$ ture, 405, 183-187.

11) Hill, E. L., Turner, R. and Elde, R. (1991) Effects of neonatal sympathectomy and capsaicin treatment on bone remodeling in rats. Neuroscience, $\mathbf{4 4 ,} 747-$ 755.

12) Adam, C., Llorens, A., Baroukh, B., Cherruau, M. and Saffar, J. L. (2000) Effects of capsaicin-induced sensory denervation on osteoclastic resorption in adult rats. Exp. Physiol., 85, 62-66.

13) Kusano, K., Miyaura, C., Inada, M., Tamura, T., Ito, A., Nagase, H., Kamoi, K. and Suda, T. (1998) Regulation of matrix metalloproteinases (MMP-2, -3, -9 , and -13) by interleukin- 1 and interleukin- 6 in mouse calvaria: association of MMP induction with bone resorption. Endocrinology, 139, 1338-1345.

14) O'Connell, P. J., Pingle, S. C. and Ahern, G. P. (2005) Dendritic cells do not transduce inflammatory stimuli via the capsaicin receptor TRPV1. FEBS Lett., 579, 5135-5139.

15) D'Souza, S. M., MacIntyre, I., Girgis, S. I. and Mundy, G. R. (1986) Human synthetic calcitonin gene-related peptide inhibits bone resorption in vitro. Endocrinology, 119, 58-61.

16) Zaidi, M., Fuller, K., Bevis, P. J., GainesDas, R. E., Chambers, T. J. and MacIntyre, I. (1987) Calcitonin gene-related peptide inhibits osteoclastic bone resorption: a comparative study. Calcif. Tissue Int., 40, 149-154.

17) Kim, C. S., Kawada, T., Kim, B. S., Han, I. S., Choe, S. Y., Kurata, T. and Yu, R. (2003) Capsaicin exhibits anti-inflammatory property by inhibiting I $\kappa \mathrm{B}-\mathrm{a}$ degradation in LPS-stimulated peritoneal macrophages. Cell. Signalling, 15, 299-306.

18) Chen, C. W., Lee, S. T., Wu, W. T., Fu, W. M., Ho, F. M. and Lin, W. W. (2003) Signal transduction for inhibition of inducible nitric oxide synthase and cyclooxygenase- 2 induction by capsaicin and related analogs in macrophages. Br. J. Pharmacol., 140, 1077-1087.

19) Sancho, R., Lucena, C., Macho, A., Calzado, M. A., Blanco-Molina, M., Minassi, A., Appendino, G. and Munoz, E. (2002) Immunosuppressive activity of capsaicinoids: capsiate derived from sweet peppers inhibits NF- $\kappa \mathrm{B}$ activation and is a potent antiinflammatory compound in vivo. Eur. J. Immunol., 32, 1753-1763. 